\title{
BESPRECHUNGSAUFSATZ
}

\section{Zur Zusammenarbeit von Völkerrechts- und Sozialwissenschaft am Beispiel des Konfliktes im Horn von Afrika*}

Zusammenarbeit von Völkerrechtlern und Sozialwissenschaftlern, die sich mit internationaler Politik befassen, ist im Rahmen von Äußerungen zu Methodenfragen ein allenthalben anzutreffendes Postulat. Dies gilt jedenfalls für die völkerrechtliche Seite ${ }^{1}$, während der Geringschätzung, die das Phänomen Recht unter Sozialwissenschaftlern im allgemeinen erfährt, eine solche des Völkerrechts im besonderen entspricht. Als einer „Formalie“ billigt man ihm wenig Bedeutung zu, hält es folglich für eine bloße Verkleidung, eine Präsentationsweise des sog. Materialen, eben der Fakten, und ist geneigt, diese Trennung für eine solche zwischen Unwichtigem und Wichtigem zu halten. Dabei finden sich kaum Äußerungen im Schrifttum, die dies in ähnlicher direkter Manier aussprechen ${ }^{2}-$ Nichtbeachtung völkerrechtlicher Fragestellungen, die sich sogar in fehlenden Fußnotenverweisen dokumentiert, ist die Regel ${ }^{3}$. Während heute so manche völkerrechtliche Arbeit fleißig auf den Tatsachenhintergrund ihres Gegenstandes verweist und hier und da auch ein politikwissenschaftliches Werk zu Rate zieht, widerfährt dem Völkrerechtler selten genug eine Berücksichtigung seiner Bemühungen im sozialwissenschaftlichen Schrifttum ${ }^{4}$. Damit sei nicht behauptet, daß dem Bekenntnis zu dem eingangs erwähnten Postulat des völkerrechtlichen methodologischen Schrifttums auch immer oder gar in ausreichendem Maß Taten folgten, die es einlösten. Wie und mit welchem Nutzen dies geschehen kann, ist auch durchaus noch nicht theoretisch abgeklärt ${ }^{5}$. Als bloße Forderung, das Völkerrecht nicht als isolierte Erscheinung in den internationalen Beziehungen aufzufassen, nicht von den Fakten und Entwicklungen zu abstrahieren, zu deren Regelung es Aussagen trifft und die wiederum seine Inhalte beeinflussen, wäre das Postulat im Grunde

* Zugleich eine Besprechung von Volker Matthies, Der Grenzkonflikt Somalias mit Äthiopien und Kenya. Analyse eines zwischenstaatlichen Konfliktes in der Dritten Welt, Hamburger Beiträge zur Afrika-Kunde, Band 21, 1977, XIII, 478 Seiten, 50.- DM.

1 Stellvertretend für viele Kimminich, Einführung in das Völkerrecht, 1975, S. $29 \mathrm{ff}$.; Weber/von Wedel, Grundkurs Völkerrecht, 1977, S. 20 f., dazu die Besprechung VRƯ 11 (1978), S. 130 ff.; s. auch Abendroth, in: Krippendorff (Herausg.), Internationale Beziehungen, 1973, S. 16 f.

$2 \mathrm{Als}$ Beispiel vgl. L.-R. Reuters Rezension des Werkes von Dicke/Rengeling, Die Sicherung des Weltfriedens durch die Vereinten Nationen. Ein Ưberblick über die Befugnisse der wichtigsten Organe, 1975 (PVS 18, 1977, S. $100 \mathrm{ff}$; S. 102: „Eine Analyse der Friedenssicherungsbefugnisse der Organe der Vereinten Nationen bleibt formalistisch und wirklichkeitsfremd, wenn nicht die Institution selbst in vergangenen Konflikten und in ihrer gegenwärtigen Situation zugleich Gegenstand sozialwissenschaftlicher Betrachtung ist.").

3 Als Beispiel diene der Beitrag von Steinhaus, in: Krippendorf (Herausg.), Probleme der internationalen Beziehungen, 1972, S. $159 \mathrm{ff}$. über "Koloniale Revolution und militärische Intervention“, der die heftig diskutierten völkerrechtlichen Probleme militärischer Interventionen in kolonialen und ehemals kolonialen Gebieten nicht erwähnt; K. Kaiser, selbst Sozialwissenschaftler, konstatiert „schiere Ignoranz dessen, was auf dem Gebiet der Völkerrechtswissenschaft geschieht" als regelmäßig anzutreffende Einstellung im Fach Internationale Beziehungen (FW 58, 1975, S. 203).

4 Nur am Rande sei vermerkt, daß das Völkerrecht auch in die allgemeine methodische und rechtstheoretische Diskussion unter Juristen nur sehr selten einbezogen wird: Weder die als Standardwerk geltende Methodenlehre von Larenz (3. Aufl., 1975) noch eine neuere "Einführung in die Rechtsphilosophie und Rechtstheorie der Gegenwart“ (Kaufmann/Hassemer, Hrsg.; 1977) befassen sich mit Völkerrecht, s. aber H. L. A. Hart, The Concept of Law, 1961 (deutsche Ausgabe 1973), Kap. X.

5 Diese Feststellung gilt auch für das Verhältnis von Rechts- und Sozialwissenschaften, vgl. Schünemann, Sozialwissenschaften und Jurisprudenz, 1976, S. 2 und öfter; s. auch K. Lenk, VRÜ 9 (1976), S. 139 grundlegend zum Verhältnis Völkerrechts- und Sozialwissenschaften im deutschsprachigen Schriftum Simma, OZ̈̈R 23 (1972), S. $293 \mathrm{ff} . ;$ Kimminich, ArchVR 16 (1974/75), S. $129 \mathrm{ff} . ;$ Czempiel, FW 58 (1975), S. $55 \mathrm{ff}$; Delbrück, in: Grimm (Herausg.), Rechtswissenschaft und Nachbarwissenschaften, Band 2, 1976, S. $79 \mathrm{ff}$;; Krakau, ebenda, S. $192 \mathrm{ff}$., alle m. w. Nachw.; vgl. ferner die bei Neuhold, Internationale Konflikte - verbotene und erlaubte Mittel ihrer Austragung, 1977, S. 2 ff. nachgewiesene Literatur; s. auch den Tagungsbericht von Wege, PVS 14 (1973), S. $140 \mathrm{ff}$. 
banal6. Kaum ein völkerrechtlicher Autor wird genannt werden können, der sich einer solchen Forderung bewußt verschlossen hätte. Wenn dies aber so ist, dann kann das Unbehagen der Sozialwissenschaftler am Völkerrecht nicht durch bloße Bereitschaft zur Berücksichtigung sog. außerjuristischer Sachverhalte abgebaut werden - soweit es Juristen überhaupt möglich ist, den Abbau dieses Unbehagens zu bewirken.

Die Forderung, sozialwissenschaftliche Fragestellungen und sozialwissenschaftlich gefundene Arbeitsergebnisse zu berücksichtigen, reicht jedoch weiter. Es genügt nicht, den tatsächlichen Hintergrund von Normen lediglich zu illustrieren, also sozialwissenschaftliche Erkenntnisse schlicht $\mathrm{zu}$ nennen und unverbunden neben den juristischen Befund zu stellen, wie dies gelegentlich geschieht. Information über die internationale soziale Wirklichkeit kann und muß vielmehr dazu genutzt werden, mehr Klarheit über den Inhalt von Normen zu gewinnen. Dies gilt zum einen für das Völkergewohnheitsrecht. Der Entstehungsprozeß gewohnheitsrechtlicher Normen sowie ihr stetiger, den Veränderungen der Realität folgender Wandel ist ohne Ausleuchtung der beteiligten Interessen und empirische Klärung der sie manifestierenden Handlungen nicht $\mathrm{zu}$ verstehen. Gleiches gilt für zahlreiche vertraglich vereinbarte Normen, über deren Inhalt kein oder kein leicht erkennbarer Konsens besteht (man denke nur an die Regeln der friedlichen Koexistenz). Erhebt man gegenüber einer Zusammenarbeit von Völkerrechts- und Sozialwissenschaft den Anspruch, zu diesem Berührungspunkt der Disziplinen genaue Ergebnisse vorzulegen, so ist der Stand der Zusammenarbeit zum gegenwärtigen Zeitpunkt noch nicht befriedigend.

Bei der eingangs konstatierten Unlust der meisten Politikwissenschaftler, völkerrechtliche Fragestellungen zu berücksichtigen oder auch nur auf sie hinzuweisen, ist ein Werk wie die jüngst erschienene Studie von Volker Matthies über die Grenzkonflikte im Horn von Afrika besonders zu begrüßen. Es hat hier ein Politikwissenschaftler, der sich der Friedens- und Konfliktforschung (S. 15 ff.) sowie einer sozialwissenschaftlich orientierten Afrika-Forschung (S. III) verbunden fühlt, in einem „eklektischen Verfahren“ (S. 24) Historie, Geographie und Sozialanthropologie (ebenda) in seine Forschung einbezogen, Ethnosoziologie und Jurisprudenz beachtet (S. 14). Inwieweit diese Arbeit dem Völkerrechtler nützen kann und welche Probleme die Methodenvielfalt aufwirft, soll im folgenden angesprochen werden. Dabei seien die folgenden Aspekte in den Vordergrund gestellt: Zum einen fragt sich, ob die Arbeit durch ihre Schilderung der Praxis klärend zur Interpretation völkerrechtlicher Normen beiträgt, zum anderen interessiert, ob die vom Sozialwissenschaftler selbst unternommenen völkerrechtlichen Subsumtionen stimmig sind, schließlich, ob er den Stellenwert, die Effektivität des Rechts in dem konkreten Konflikt zutreffend w ürdigt ${ }^{7}$.

Die erste Frage ist bei der Untersuchung eines Geschehens in der Dritten Welt auch deshalb besonders wichtig, weil die dortigen Vorgänge in der Regel weniger gut durch die üblichen Quellen dokumentiert sind als solche in Europa oder Amerika: Materialien über Staatenakte, insbesondere deren innenpolitischen Hintergrund, werden kaum regelmäßig publiziert, eine kritische Presse ist nur in wenigen Staaten vorhanden. Der Völkerrechtler bedarf also der politikwissenschaft-

6 Das Bild vom Einrennen offener Türen verwendet Randelzhofer, FW 58 (1975), S. 252 f.; vgl. auch Young, AJIL 66 (1972), S. 61.

7 Dazu Simma, FW 57 (1974), S. 81; K. Kaiser, FW 58 (1975), S. 199 ff. 
lichen Aufbereitung der Fakten in besonderem Maße, kann sich nicht in der ihm gewohnten Weise direkt an Gesetzblätter, offizielle Bulletins und private Dossiers halten. Außerdem ist die Untersuchung von internationalen Auseinandersetzungen in der Dritten Welt, und hier insbesondere in Afrika und Asien, noch immer Stiefkind der Völkerrechtswissenschaft. Zwar ist erkannt worden, daß die gegenwärtige Struktur der internationalen Beziehungen, die den Schwerpunkt der Völkerrechtserzeugung auf die Vereinten Nationen verlegt hat, wegen des dort zahlenmäßig großen Gewichts der Staaten aus der Dritten Welt deren Auffassungen und Praxis zu den Kernfragen des modernen Völkerrechts berücksichtigen muß. Die Untersuchungen, die diesem Gegenstand gewidmet sind, präsentieren aber bisher zumeist überblickartige Zusammenfassungen ${ }^{8}$ und widmen sich kaum in monographischer Weise Einzelfragen ${ }^{9}$. Bei völkerrechtlichen Untersuchungen von Geschehnissen in der Dritten Welt kann sich eine Kooperation von Völkerrechts- und Sozialwissenschaft in besonderer Weise bewähren, denn wegen der vielfach nachzuweisenden Abweichungen dieser Staaten von traditionellem Völkerrecht ist überhaupt nur Klarheit über die jeweilige Rechtslage zu gewinnen, wenn die völkerrechtlichen Normen aus ihrer Wirkungsweise in der sozialen Wirklichkeit heraus interpretiert werden. Die Praxis und Theorie der Staaten der Dritten Welt gibt wichtige Auskünfte über das dort aktuell geltende Völkerrecht; es sind nicht schlicht Fakten an Normen zu messen, sondern die Fakten müssen bereits betrachtet werden, um die Normen erst erkennen zu können: ob die Staaten der Dritten Welt ohne ihre Mitwirkung entstandenes Gewohnheitsrecht pauschal oder teilweise übernommen, ob sie partikulares, allein auf ihre Beziehungen untereinander anwendbares Recht geschaffen haben oder im Begriff sind, solches Recht zu schaffen - diese Fragen sind in jedem Einzelfall nur mit Hilfe genauer Analyse der Staatenpraxis zu beantworten.

Die Frage nach korrektem Umgang mit völkerrechtlichen Begriffen interessiert den Völkerrechtler zunächst ganz vordergründig wie jeden, der prüft, wie s e in Geschäft durch andere besorgt wird. Daneben aber tritt der folgende - wichtigere Gesichtspunkt: Interdisziplinäre Zusammenarbeit kann nur in Ausnahmefällen bedeuten, daß ein Wissenschaftler die Arbeit mehrerer Disziplinen leistet, deren methodisches Vorgehen also gleichsam in einer Person integriert, wie das jüngst etwa Neuhold unternommen hat ${ }^{10}$. Der Regelfall wird vielmehr sein müssen und das nicht zuletzt wegen der gegenwärtig bestehenden und auch künftig eher zum Methodenmonismus tendierenden Ausbildungsstruktur -, daß die Arbeit mehrerer Fachdisziplinen erst das interdisziplinäre Ganze ausmacht. Eine solche Zusammenarbeit ist aber nur dann gedeihlich, wenn "Grenzüberschreitungen " von beiden Seiten mit der gebotenen Zurückhaltung vonstattengehen oder aber auf gediegener Kenntnis beruhen. Im Interesse der Sache sollte daher eine gegenseitige kritische Beobachtung stattfinden.

Die voluminöse Studie von Matthies enthält 478 ungewöhnlich eng bedruckte Seiten und ist dem in der zweiten Jahreshälfte 1977 und Anfang 1978 Schlagzeilen machenden Grenzkonflikt zwischen Somalia und Äthiopien sowie dem derzeit mehr schwelenden als lodernden Grenzkonflikt zwischen Somalia und Kenia ge-

8 Z. B. D. Schröder, Die Dritte Welt und das Völkerrecht, 1970; Anand, New States and International Law, 1972.

9 Eine völkerrechtliche Untersuchung des Angola-Konfliktes hat jüngst Bothe vorgelegt (ZaöRV 37, 1977,

10 Internationale Konflikte - verbotene und erlaubte Mittel ihrer Austragung, 1977. 
widmet $^{11}$. Das Thema ist nicht nur ein Tagesthema, kann man doch vom Fortgang der Ereignisse im Horn von Afrika, dem nach Angola zweiten Schauplatz sow jetischer und kubanischer Militärpräsenz in Afrika, tiefgreifende Auswirkungen auf die künftige Afrikapolitik der Großmächte, aber auch die Chancen einer afrikanischen Integration erwarten. Auch ist der Typus Grenzkonflikt im innerafrikanischen Geschehen sehr weit verbreitet (man denke an jüngere oder noch anhaltende Auseinandersetzungen zwischen Libyen und dem Tschad, Benin und Togo, Mali und Obervolta, Uganda und Tansania, Äthiopien und dem Sudan, Lesotho und Südafrika) ${ }^{12}$. Im übrigen hebt Matthies zurecht hervor, daß die Untersuchung gerade dieses Konfliktes Erkenntnisse erbringt, die eine Typologie zwischenstaatlicher Konflikte in der Dritten Welt vielleicht nicht schon ermöglichen, ihr aber doch vorarbeiten können (vgl. S. 23 ff.). Insbesondere die Kriegsursachenforschung wäre hierdurch bereichert. Schließlich ist auch die völkerrechtliche Relevanz des Themas, um die es in dieser Betrachtung vor allem gehen soll, evident. Welche völkerrechtlichen Fragen der Konflikt im Horn aufwirft, wird weiter unten im einzelnen zu erörtern sein.

Matthies' Arbeit beginnt mit einer methodologischen, die Vielschichtigkeit der hier gebotenen Vorgehensweise hervorhebenden Einleitung, die sich zu einer „peripheren" Betrachtungsweise bekannt ${ }^{13}$. Sie wendet sich sodann der „Aitiologie und Genesis des Konflikts" zu, schildert die koloniale Expansion Großbritanniens und Italiens sowie den hierauf reagierenden Somali-Nationalismus, der sich bereits in den ersten Jahren dieses Jahrhunderts in organisierten Widerstandsbewegungen manifestierte. Ausführlich werden die Komponenten des Somali-Nationalismus, vor allem seine religiösen Züge analysiert, seine politische Entwicklung wird bis zur Gründung der Republik Somalia im Jahre 1960 auf den Territorien von Italienischund Britisch-Somalia verfolgt. Das Bild Somalias als Verlierer der kolonialen Epoche wird deutlich abgehoben von den insoweit "glücklicheren" Äthiopien (mit seinen traditionell intensiven Beziehungen zur übrigen afrikanischen Staatenwelt) und Kenia, das von ökonomischen und strategischen Interessen des Vereinigten Königreichs profitierte. Ein drittes Kapitel gibt sodann die „Positionen“ der drei Konfliktparteien wieder, die es den Stichworten "territorialer Revisionsmus“, „Selbstbestimmung“ bzw. „territorialer Status quo“, „Selbsterhaltung“ zuordnet; offizielle und halboffizielle Verlautbarungen von Vertretern Somalias, Aithiopiens und Kenias werden hier ausführlich dokumentiert. „Drohpolitik“ sowie „Gewalt und Krieg" nennt Matthies die nun folgenden, ins Einzelne gehenden Beschreibungen der Aktionen der drei Staaten von Verhandlungen über Propagandakrieg und Rüstungswettlauf bis zu Grenzscharmützeln.

Bei der Untersuchung möglicher innenpolitischer Determinanten des Konflikts arbeitet Matthies überzeugend die Unterschiede zwischen einem ethnisch und kulturell homogenen Nationalstaat (Somalia) und den weitgehend ohne solche Bindung auskommenden Staatsgebilden Kenia und Äthiopien heraus, die gezwungen sind, jedem somalischen Begehren nach territorialen Zugeständnissen hartnäckig zu wehren: Veränderungen des Status quo könnten (vor allem in Äthiopien)

11 Die Probleme um das 1977 als Djibouti unabhängig gewordene Territorium der Afar und Issa klammert Matthies aus, S. 30, Anm. 37.

$12 \mathrm{Vgl}$. Oye Cukwurah, IJIL 13 (1973), S. $176 \mathrm{ff}$; Cervenka, VRU 7 (1974), S. $126 \mathrm{ff}$. m. w. Nachw. "Staaten, Gesellschaften und Menschen" in der Dritten Welt in den Mittelpunkt der Untersuchung rückt (vgl. S. 2 ff.). 
einen Domino-Effekt auslösen. Kapitel 7 erörtert die internationalen Bestimmungsfaktoren des Konfliktes. Es stellt ihn in die Perspektive der Ost/West-Auseinandersetzungen, würdigt die Rolle der Organisation für afrikanische Einheit (OAU), die seit ihrer Gründung mit dem Konflikt konfrontiert war, sowie den Einfluß der arabischen Staaten auf das Konfliktgeschehen, insbesondere des benachbarten Sudans und Ägyptens. In teilweiser Überschneidung $\mathrm{zu}$ diesem Kapitel stellt Matthies dann die bi- und multilateralen Bemühungen um eine Beilegung der Auseinandersetzungen zusammenfassend dar. Die Schlußkapitel 9 und 10 schließlich liefern ein politikwissenschaftlich-theoretisches Resümee sowie eine gestraffte 24seitige Darstellung „aktueller Perspektiven“. Dem von der Tagesaktualität der Geschehnisse im Horn von Afrika zu dem Werk hingeführten Interessenten sei gesagt, daß dieses seinen „zeitlichen Schwerpunkt“ auf die Jahre zwischen 1960 und 1970 legt (S. III), jüngere Fakten und Literatur sind aber allenthalben berücksichtigt. Dünner wird dieser Zustrom nur für die Zeit nach 1976. Die Arbeit wurde in der vorliegenden Form im Juli 1977 abgeschlossen.

Die thematische Breite des Buches, die hier nur oberflächlich skizziert wurde, deckt Matthies mit imponierender Gründlichkeit ab. Forschungsreisen nach Ostafrika haben ihm eine Fülle von Materialien zugänglich gemacht, die sorgfältig verwertet und in übersichtlicher und infolge der klaren Gliederung auch für nachschlagende Leser gut auffindbarer Weise dargeboten werden. Karten, Tabellen und graphische Darstellungen finden sich in großer Zahl. Aus diesen Feststellungen folgt bereits, daß der Arbeit die erste oben als für den Völkerrechtler interessant bezeichnete Qualität - Verwendbarkeit als Materialquelle - in hohem Maße eignet. Die hervorragende Faktenverwertung und überzeugende theoretische Analyse macht das komplizierte Geschehen transparent, hebt die Entwicklungslinien deutlich hervor, bereitet damit die Staatenpraxis in einer Weise auf, die der Völkerrechtler selbst nicht oder kaum in zulänglicher Weise leisten könnte ${ }^{13 a}$. Dem, der den Konflikt Somalias mit seinen Nachbarn völkerrechtlich untersuchen will, ist mit Matthies' Arbeit ein ausgezeichneter Dienst getan. Auch wer sich über Praxis und Theorie afrikanischer Staaten zu völkerrechtlichen Fragen ein Bild machen will, wird mit Nutzen zu ihr greifen.

Nun soll sich aber - wie angedeutet - interdisziplinäre Zusammenarbeit nicht auf - wechselseitig denkbare - Dienstleistung beschränken. Die zweite oben angesprochene Frage sei daher wieder aufgenommen: Überzeugt die Arbeit auch, soweit sie völkerrechtliche Untersuchungen anstellt bzw. die Rolle des Völkerrechts im Konflikt anspricht? Erste ins Blickfeld gelangende völkerrechtliche Frage bei Gebietskonflikten ist die nach der territorialen Zugehörigkeit des umstrittenen Gebietes. Anders als bei den europäischen Grenzen, die sich als Abgrenzungen von $S \mathrm{t}$ a a $\mathrm{ts}$ gebie t über Jahrhunderte zurückverfolgen lassen, wurde eine juristisch faßbare territoriale Aufteilung vieler afrikanischer Gebiete erst durch die koloniale Expansion ausgelöst. Als das Vereinigte Königreich, Frankreich und Italien das Horn von Afrika erreichten, begegneten ihnen nicht geschlossene Staatswesen, sondern eine Vielzahl selbständiger Clans sowie - in Küstennähe von Türken, Ägyptern und Sansibar-Arabern verwaltete Stadtstaaten. Mit zahlreichen der Clans schlossen die europäischen Mächte Protektoratsverträge ab: es

13a Der von einem Rezensenten erhobene Vorwurf, Matthies habe lediglich eine "Chronik" geliefert, ohne seinen theoretischen Anspruch einzulösen (D. Karsten, Zeitschrift für Kulturausstausch 28 [1978], S. $9 \mathrm{f}$.), ist durchaus unbegründet. 
entstanden Französisch- (1885), Britisch- (1887) und Italienisch-Somalia (1908). Zwischen 1888 und 1910 schlossen die europäischen Mächte untereinander und mit Äthiopien Grenzverträge ab. Im Jahre 1925 trat Großbritannien des Juba-Land an Italien ab; 1934, nach der Besetzung Äthiopiens, ordnete Italien den Ogaden Somalia als einer Verwaltungseinheit innerhalb von Italienisch-Ostafrika zu. Nach Verträgen aus den Jahren 1942 und 1944 wurde schließlich der britisch-äthiopische Vertrag von 1954 abgeschlossen, der Äthiopien die Herrschaft über den Ogaden einräumt, zugleich aber dort Weiderechte für die nomadisierenden Somali vorsieht.

Matthies referiert die hier angesprochenen Ereignisse und Verträge (ohne deren Texte immer nachzuweisen); seine Auswertung befriedigt aber nicht immer. Die inhaltliche Darstellung und die Untersuchung, ob die Verträge heute noch völkerrechtlich verbindlich sind, wird getrennt - letzteres bei der Vorstellung der "Positionen“ der Konfliktparteien mitbehandelt. Diese Vorgehensweise verstellt den Blick auf den Unterschied zwischen politischen und rechtlichen Ansprüchen: Ein Satz wie „Somalia hielt die gesamte kolonial vererbte territoriale Allokation und Grenzziehung für ,illegitim', ,unmoralisch' und völkerrechtlich ungültig, weil sie von imperialistischen fremden Mächten gegen den Willen bzw. ohne die Zustimmung der betroffenen Somali-Bevölkerung vorgenommen worden sei“ (S. 106) ist zu undifferenziert.

Zudem wird die Position Äthiopiens zur Frage der Weitergeltung der Verträge nach der somalischen Unabhängigkeit von 1960 in zumindest mißverständlicher Verkürzung wiedergegeben. Äthiopien hat jedenfalls zunächst nicht pauschal die Weitergeltung des Vertrages von 1954 behauptet und sich hinsichtlich der Weiderechte für "nicht unbedingt" verpflichtet gehalten (so aber Matthies, S. 116 f.). Vielmehr hat die äthiopische Regierung offiziell erklärt, sie halte den Vertrag wegen der Erlangung der Unabhängigkeit durch Somalia für „automatically invalid"14. Daß hier wohl nur partielle Ungültigkeit gemeint - jedenfalls angestrebt - war, kann aus der Erklärung unmittelbar nicht entnommen, höchstens aus den Begleitumständen und anderen Äußerungen erschlossen werden ${ }^{15}$.

Einer klaren Herausarbeitung dieser Frage bedarf es, weil die völkergewohnheitsrechtlich geltenden Regeln über die Nachfolge von Staaten in Verträge überhaupt erst dann praktisch werden, wenn nur einer der beiden Kontrahenten Weitergeltung des Vertrages behauptet oder bestreitet. Denn auch stillschweigende Vertragsauflösung wäre denkbar; diese könnte sich unter Umständen nur auf einen Teil der Verträge erstrecken.

Erörtert werden müßte ferner, ob die Weiderechte radizierte Rechte sind, also gebietsbezogen, oder ob sie nur dem ursprünglichen Vertragspartner eingeräumt werden sollten ${ }^{16}$.

Die Festschreibung der kolonialen Grenzen durch Art. III Nr. 3 (s. auch Art. II Nr. 1 c) der Charta der OAU17 wird von Matthies nicht im Zusammenhang mit der Gültigkeit der Grenzen erörtert, sondern im Kapitel über die „Internationalen Beziehungen der Kontrahenten" (S. 325 ff.). Die Frage, ob Somalia durch seinen Beitritt zu der Organisation sich der Möglichkeiten begeben hat, seine Grenzen mit Äthiopien und Kenia mit völkerrechtlichen Argumenten anzugreifen, wird

14 The Times, 6. Juni 1960.

15 S. dazu Brown, ICLQ 10 (1961), S. 169 f.

16 Auch dazu Brown, ebenda, S. $172 \mathrm{f}$.

17 Vom 13. September 1963; UNTS Vol. 479, S. $39 \mathrm{ff}$. 
daher nicht aufgeworfen. Die Resolution „Border Disputes among African States“, verabschiedet während der OAU-Gipfelkonferenz in Kairo 196418, wird von Matthies offenbar für wichtiger gehalten als die OAU-Charta selbst (vgl. S. 327 ff.), obwohl diese ein echter Vertrag ist, Resolutionen der Versammlung der Staatsoberhäupter und Regierungschefs dagegen jedenfalls nach der Charta keinen bindenden Charakter haben (vgl. Art. VIII bis XI). Die Normativität der Resolution wird nicht untersucht - typisch für eine politikwissenschaftliche Vorgehensweise, der es auf den politischen, nicht den juristischen Wert einer Willensbekundung von Staaten ankommt - er ist in der Tat bei formlosen Absichtserklärungen gelegentlich größer als bei Verträgen. Schließlich hätte auch untersucht werden können, ob das afrikanische Tabu der kolonialen Grenzziehung nicht in neuerer Zeit wieder in Frage gestellt worden ist (und dies auch mit juristischen Konsequenzen): Der Informationsminister von Togo beispielsweise hat es im Januar 1976 anläßlich einer Grenzkorrektur zwischen Gambia und dem Senegal als Anachronismus bezeichnet ${ }^{19}$; Präsident Amin von Uganda richtete im Februar des gleichen Jahres territoriale Ansprüche an Kenia und den Sudan ${ }^{20}$. Die Untersuchung der juristischen Legitimität des territorialen Status quo im Horn von Afrika wird somit von Matthies insgesamt nicht soweit vorangetrieben, daß ein Ergebnis erzielt würde ${ }^{21}$.

An die Beurteilung der Gültigkeit der Grenzen schließt sich für den Völkerrechtler die Frage an, ob eine der beteiligten Konfliktparteien einen völkerrechtlichen Anspruch auf Veränderung dieser Grenzen hat. Ein solcher Anspruch könnte hergeleitet werden aus dem Selbstbestimmungsrecht. Der Gedanke der Selbstbestimmung ist in der Charta der Vereinten Nationen angesprochen (Art. 1 Nr. 2; 55), in seiner gegenwärtigen Ausgestaltung wird er vor allem durch Deklarationen der Generalversammlung der Vereinten Nationen geprägt ${ }^{22}$. Seine Vagheit und seine enge Verbindung zu rein politischen Ansprüchen erschweren den juristischen Umgang mit dieser Materie ${ }^{23}$. In der Argumentation der Somali spielt das Selbstbestimmungssrecht eine wichtige Rolle; Matthies befaßt sich eingehend mit Inhalt und Bedeutung dieser Argumentation (durchgängig in Kapitel 3). Sein Überblick über die hier anzustellenden juristischen Erwägungen läßt aber die Fragestellung nicht klar werden. Den Kern des Problems sieht Matthies in der Gegenüberstellung des Rechts auf Selbstbestimmung und des „Rechts der Staaten auf Selbsterhaltung " (das Äthiopien und Kenia geltend machten, S. $122 \mathrm{ff}$.) - diese Rechte schlössen einander aus (S. 121). Ergebnis ist: „Die Normen und Regeln des Völkerrechts und internationalen Systems begünstigen daher zweifellos eher die äthiopische und kenianische Position des territorialen Status quo als die Position des territorialen Revisionismus der Republik Somalia." Abgesehen von der mangelnden Präzision dieser Feststellung (wie stehen hier Normen und Regeln zueinander, wie Völkerrecht und internationales System dazu und zueinander?) sagt sie als Ergebnis zu wenig aus. Dies resultiert aus der erwähnten Gegenüberstellung von Selbstbestimmung und Selbsterhaltung, die zu einer verfehlten Blickrichtung

18 AHG/Res. 16 I; Text bei Brownlie, Basic Documents of African Affairs, 1971, S. $360 \mathrm{f}$.

19 Africa Research Bulletin, S. 3889 f. (Jan. 1976).

$20 \mathrm{Vgl}$. Rousseau, RGDIP 80 (1976), S. $1264 \mathrm{ff}$.

21 Vgl. dazu die eingehende, inzwischen freilich veraltete Untersuchung von Brown, ICLQ 5 (1956),

$22 \mathrm{Sg}$. $245 \mathrm{ff}$. die bei Krakau/v. Wedel/Göhmann, UN-General Assembly Resolutions, 1975, S. $104 \mathrm{ff} . \mathrm{abge}$. druckten Dokumente; s. ferner Brownlie, Principles of Public International Law, 2. Aufl., 1973, S. 575 f.

23 Das stellt Matthies zu Recht fest, S. $123 \mathrm{f}$. 
führt. Nicht zwei Rechte stehen hier unvereinbar und mit Ausschließlichkeitsanspruch einander gegenüber wie Scylla und Charybdis. Das Recht auf Selbsterhaltung berechtigt nach Völkergewohnheitsrecht zur Verteidigung gegenüber Angriffen auf die territoriale Integrität ${ }^{24}$; solche sind jedoch nicht Gegenstand der überwiegend um verbale „Übergriffe“ kreisenden Debatten zwischen den Kontrahenten. Auch Art. 51 der Satzung der Vereinten Nationen hat enge tatbestandliche Voraussetzungen, die hier nicht vorliegen ${ }^{25}$. Das Prinzip der Nichteinmischung in innere Angelegenheiten ist in Art. 2 Nr. 7 der Satzung der Vereinten Nationen nur für das Verhältnis der Organisation zu ihren Mitgliedern, nicht für das Verhältnis dieser untereinander, vorgesehen (s. dazu S. 122 bzw. S. 124). Das „Recht auf Selbsterhaltung" hat nicht in Art. 2 Nr. 4 derselben Satzung "Niederschlag gefunden“ (S. 126), sondern - mit einschränkenden Modifizierungen früheren Gewohnheitsrechtes in Art. 51. Statt Rechtsnormen und politische Schlagworte, deren jeweiliger völkergewohnheitsrechtlicher oder durch Verträge festgeschriebener normativer Hintergrund nicht deutlich wird, unverbunden gegeneinander $\mathrm{zu}$ stellen, wäre zu fragen gewesen, ob geltendes universelles oder partikulares Völkerrecht ein Selbstbestimmungsrecht enthält, das seinem Träger den Anspruch verleiht, auch gegen bestehende Grenzen sich in eigener Staatlichkeit zu verwirklichen (oder sich an ein bestehendes Staatswesen anzuschließen). Ein solches Recht schlösse ein entgegenstehendes Recht auf Erhaltung aus: Die "faktische“ Einbuße an territorialer.Integrität wäre in jenem Fall keine rechtliche.

Wer Rechtsträger ist (ein Staat, ein Volk - in welcher Definition?), wer das Recht geltend machen kann (nur die in dem betreffenden Raum lebenden Nomaden selbst?), ob normative Grundlagen für eine friedliche Durchsetzung des Anspruches bestehen ${ }^{26}$, wäre zu erwägen. Die Zuordnung des somalischen Begehrens zum Dekolonialisierungsprozeß, die Somalia von Anfang, an vorgenommen hat, hätte mehr als einen Hinweis verdient (S. 124), denn die Normqualität des Anspruchs auf Dekolonialisierung ist möglicherweise eine andere als die eines allgemeinen Selbstbestimmungsrechtes ${ }^{27}$, seine Inhalte sind wegen der Deklaration der Generalversammlung der Vereinten Nationen vom 14. Dezember 1960 und der sich an ihr orientierenden Staatenpraxis konkreter.

Neben Validität der Grenzen und denkbarem Anspruch auf ihre Veränderung sind das Verhalten der Konfliktparteien selbst sowie das Verhalten dritter Staaten und internationaler Organisationen von völkerrechtlichem Interesse. Auch hier könnte zunächst gefragt werden, ob aus der Nichtzugehörigkeit von Somalia, Äthiopien und Kenia zu der Staatengruppe, die die Mehrzahl der völkerrechtlichen Regeln durch ihre Praxis hervorgebracht hat, folgt, daß die Staaten nicht an alle dieser Regeln gebunden sind oder ob partikulare Modifikationen vorliegen. Akte wie die finanzielle und militärische Unterstützung der Republik Somalia für die Westsomalische Befreiungsfront und die Somali im Norden Kenias, subversive Tätigkeit, Drohung mit Gewalt, schließlich der Einmarsch in Äthiopien selbst, könnten das

\footnotetext{
24 Statt vieler Berber, Lehrbuch des Völkerrechts, Band 1, 2. Aufl., 1975, S. 195 ff.

25 S. z. B. Skubiszewski, in: Sørensen (Herausg.), Manual of Public International Law, 1968, S. $765 \mathrm{ff}$.

$26 \mathrm{Vgl}$. die Ưberlegungen, die Weber über die Möglichkeiten der friedlichen Beilegung einer anderen Gebietsstreitigkeit in der Dritten Welt anstellt ("Falkland-Islands“ oder "Malvinas“?, 1977, S. 127 ff.).

$27 \mathrm{Vgl}$. dazu z. B. Nincic, The Problem of Sovereignty in the Charter and in the Practice of the United Nations, 1970, S. $234 \mathrm{ff} . ;$ a. A. z. B. Mössner, Einführung in das Völkerrecht, 1977, S. 205 f.; im Zusammenhang mit der Deklaration vom 14. Dezember 1960 (1514-XV) übersieht Matthies, daß diese ein Selbstbestimmungsrecht von Bevölkerungsgruppen innerhalb eines nachkolonialen Staates jedenfalls dann vorsieht, wenn diese diskriminiert werden, vgl. Verdross/Simma, Universelles Völkerrecht. Theorie und Praxis, 1976, S. $254 \mathrm{f}$.
} 
Gewaltverbot des Art. 2 Nr. 4 der Satzung der Vereinten Nationen und die Charta der OAU verletzt; Deklarationen wie die über den Aggressionsbegriff ${ }^{23}$ und die Prinzipien-Deklaration von $1970^{29}$ wären heranzuziehen. Die von Somalia gezogene Parallele zwischen einer Unterstützung des „Befreiungskampfes“ im Ogaden zu solcher des Kampfes der Schwarzen gegen Rassismus im südlichen Afrika ${ }^{30}$ wäre völkerrechtlich zu würdigen.

Auch die heftige Propagandatätigkeit aller drei Seiten (hierzu S. 153 ff.) könnte völkerrechtswidrig sein. Matthies' im Anschluß an Zartman ${ }^{31}$ getroffene Feststellung, Propaganda werde in den zwischenstaatlichen Beziehungen Afrikas als Einmischung in die inneren Angelegenheiten angesehen und daher als "feindseliger Akt" gewertet (S. 153), zeigt noch einmal in nuce die Problematik von Aussagen im Grenzgebiet von Völkerrechts- und Sozialwissenschaft. Als völkerrechtlicher Befund ist die These vermutlich unzutreffend - es bedürfte des Nachweises aus der Staatenpraxis, wie hoch die Staaten die Schwelle ansetzen, bei deren Überschreitung ein Rechtsverstoß vorliegt. Da die These allein auf den afrikanischen Kontinent bezogen ist, müßten offizielle und offiziöse Äußerungen sowie die daraufhin erfolgenden Reaktionen der afrikanischen Staaten analysiert werden. Eine solche Untersuchung ist bisher nicht vorgelegt worden. Wie durch den sog. Kalten Krieg die erwähnte Schwelle im Vergleich mit dem von Empfindsamkeit in den internationalen Beziehungen geprägten 19. Jahrhundert erhöht worden ist ${ }^{32}$, so dürfte aber auch die wenig zurückhaltende Art afrikanischer Propaganda eher dazu geführt haben, daß grobe Töne hingenommen werden, völkerrechtlich irrelevant sind. Der hier skizzierte, von der Staatenpraxis auf die Norm schließende Weg völkerrechtlicher Untersuchungen kontrastiert deutlich mit der Begründung, die Matthies liefert: Die Schwäche der afrikanischen Staaten mache sie „gegenüber jeder Bedrohung ihrer Unabhängigkeit und Legitimität, und sei sie auch nur verbal, sehr empfindlich" (S. 153) - die mit Methoden der Sozialwissenschaften gefundene Bewertung (Schwäche), verleitet hier also zu einer juristischen Fehlbeurteilung. Was schließlich das Verhalten afrikanischer und außerafrikanischer Drittstaaten sowie der Vereinten Nationen, der OAU und der Arabischen Liga (der Somalia seit 1974 angehört) anlangt, so möge der Hinweis genügen, daß auch hier eine Vielzahl von völkerrechtlichen Fragen auftaucht, die um Gewalt- und Einmischungsverbot, Rechtsfragen des Bürgerkrieges und Neutralität kreisen, ihre Brisanz allerdings im wesentlichen erst durch die militärische Eskalation des Konfliktes gewonnen haben - zu einem Zeitpunkt also, der von Matthies nicht mehr einbezogen werden konnte. Bedeutungsvoll ist hier insbesondere die Frage, ob durch das Selbstbestimmungsrecht der Völker jedenfalls im antikolonialen Befreiungskampf das Verbot der Gewaltanwendung in den internationalen Beziehungen eingeschränkt wird ${ }^{33}$ : Der somalische Präsident Barre hat im Oktober 1977 die äthiopische Politik als „black power colonialism“ bezeichnet ${ }^{34}$.

28 Vom 14. Dezember 1974 (3314-XXIX), dazu z. B. Zourek AFDI 20 (1974) S. 9 ff.

29 Vom 24. Oktober 1970 (2625-XXV), dazu z. B. Neuhold, op. cit. (Anm. 10).

30 Radio Somalia laut FAZ vom 21. Juli 1977.

31 International Relations in the New Africa, 1966, S. 87, der sich allerdings nur auf westafrikanische

32 Vgl. von Münch, Das völkerrechtliche Delikt in der modernen Entwicklung der Völkerrechtsgemeinschaft, 1963, S. $74 \mathrm{ff}$.

33 Vgl. dazu die Nachweise bei Bothe, ZaöRV 37 (1977), S. 575, Anm. 16, der selbst eine entsprechende Rechtsentwicklung bezweifelt (S. 576), sowie E. Klein, ZaöRv 36 (1976), S. $618 \mathrm{ff}$.

34 Africa Research Bulletin, S. 4591 (Okt. 1977). 
Eingangs wurde die Untersuchung der Rolle, die das Völkerrecht für die Lösung internationaler Konflikte spielen kann, als wichtige Frage einer Kooperation von Sozialwissenschaften und Völkerrecht bezeichnet. Es erscheint dabei müßig, sie für eine der Disziplinen vindizieren $\mathrm{zu}$ wollen $^{35}$; beide sollten sie stellen. Matthies hat ausführlich die „Bemühungen um eine Regulierung des Konflikts“ untersucht (S. 359 ff.), dabei methodisch der Typologie von Randle ${ }^{36}$ folgend. Idealiter ergänzt werden sollte eine derartige Darstellung durch die Analyse des Stellenwertes juristischer Überlegungen im Ablauf des politischen decision making - eine freilich in Anbetracht des weitgehend unzugänglichen Materials fast utopische Forderung. Immerhin liegen einige Arbeiten vor, die bei anderen Konflikten wesentliche Einsichten dieser Art ermöglicht haben.

Die hier angestellten Überlegungen sollten auf die weithin erkannten Schwierigkeiten der Zusammenarbeit der verschiedenen Disziplinen, die sich um die Beurteilung desselben Gegenstandes Internationale Politik bemühen, an der Arbeit eines methodischer Vielfalt aufgeschlossenen Autors zu einem für alle beteiligten Disziplinen besonders interessanten Konflikt exemplifizieren. Sie legen nahe, bei Aussagen im Grenzgebiet begriffliche Vorsicht walten zu lassen und bei Überschreiten der Grenze die konkrete Zusammenarbeit mit der Nachbardisziplin zu suchen.

Politikwissenschaftliche Untersuchungen, die in dieser Weise verfahren - das illustriert die Arbeit von Matthies - werden durch die Beachtung der völkerrechtlichen Dimension Wesentliches für die Analyse eines zwischenstaatlichen Konfliktes gewinnen; sie werden darüber hinaus auch dem Völkerrechtler die Erkenntnis von Normen und ihrer Wirkungsweise erleichtern. Im Hinblick auf den Konflikt im Horn von Afrika hat Matthies auch zu der letztgenannten Aufgabe trotz der geltend gemachten Kritik Wichtiges beigetragen. Noch ertragreicher wäre Methodenvielfalt, wenn unter Sozialwissenschaftlern besser bekannt wäre, daß das Interesse der Völkerrechtswissenschaft an einem Konflikt wie dem vorliegenden weiterreicht, als dies offenbar (auch) von Matthies angenommen wird: „Ein Jurist ... nimmt eine Interpretation internationaler Grenzverträge vor und versucht, die Legitimität einer Grenze zu beurteilen“ (S. 14) - dabei sei nicht verschwiegen, daß es juristische Betrachtungen gerade auch von Grenzkonflikten gibt, die einem solchen Vorurteil Vorschub leisten.

Philip Kunig

\footnotetext{
35 Delbrück, FW 58 (1975), S. 244 f., definiert sie als solche der Sozialwissenschaften; anders Schwarzen-

berger, in: Grundprobleme des internationalen Rechts, Festschrift für Spiropoulos, 1957, S. 403.

36 The Origins of Peace. A Study of Peacemaking and the Structure of Peace Settlements, 1973.

37 Z. B. Chayes, The Cuban Missile Crisis, 1974.
} 